\title{
Urology Residency Directors' Awareness of Association between $\alpha$-Adrenergic Antagonists and Floppy Iris Syndrome and Practice Patterns Regarding Prophylactic $\alpha$-Adrenergic Antagonists
}

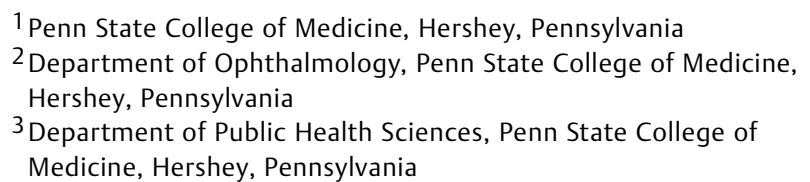

David Cui, BA ${ }^{1}$ Gaytri Gupta-Elera, MD² Ingrid U. Scott, MD, MPH 2,3

Address for correspondence Ingrid U. Scott, MD, MPH, Departments of Ophthalmology and Public Health Sciences, Penn State College of Medicine, 500 University Drive, HU19, Hershey, PA 17033-0850 (e-mail: iscott@pennstatehealth.psu.edu).

J Acad Ophthalmol 2019;11:e7-e9.

Abstract


Keywords
- cataract surgery
- ophthalmology
- urology
- intraoperative floppy

- iris syndrome
- $\alpha$ blockers
- $\alpha$-adrenergic receptor

- antagonists
- tamsulosin
- survey
- practice patterns
- residency director

Purpose The use of prophylactic $\alpha$-adrenergic receptor antagonists prior to surgery is associated with reduced postoperative urinary retention. However, $\alpha$-adrenergic receptor antagonists have been associated with intraoperative floppy iris syndrome (IFIS). Studies have shown that IFIS is correlated with higher rates of cataract surgery complications. The purpose of this study is to investigate United States urology residency program directors' (1) awareness of the association between $\alpha$-adrenergic receptor antagonists and IFIS and (2) practice patterns regarding the use of prophylactic $\alpha$-adrenergic receptor antagonists prior to surgery.

Materials and Methods An anonymous, online survey was sent to the program director of each of the 138 urology residency programs accredited by the Accreditation Council for Graduate Medical Education. The survey assessed program directors' knowledge regarding the association between $\alpha$-adrenergic receptor antagonists and IFIS, and practice patterns regarding the use of prophylactic $\alpha$-adrenergic receptor antagonists prior to surgery.

Results Sixty-nine of the 138 (50\%) residency directors responded. Forty percent of respondents believe that tamsulosin (Flomax) does not increase the risk of IFIS. Fiftytwo percent of respondents prescribe or consider prescribing prophylactic $\alpha$-adrenergic blockers prior to surgery, with tamsulosin being the most preferred $\alpha$-adrenergic blocking agent. Of the prescribing urologists, only $58 \%$ counsel most of their patients regarding the association between the prescribed drug and IFIS, $28 \%$ never ask patients whether they have had cataract surgery, and $56 \%$ would change the drug or dosage used if they learned that a patient had not yet had cataract surgery.

Conclusion The results of this study demonstrate the potential to decrease the risk of IFIS through education of urologists regarding the association of $\alpha$-adrenergic antagonists with IFIS.

(1) David Cui's ORCID is https://orcid.org/0000-0002-6101-5164.

received

March 1, 2019

accepted after revision

May 28, 2019
DOI https://doi.org/

10.1055/s-0039-1693418.

ISSN 2475-4757.
Copyright $\odot 2019$ by Thieme Medical Publishers, Inc., 333 Seventh Avenue, New York, NY 10001, USA. Tel: +1(212) 584-4662.
License terms

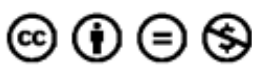


Intraoperative floppy iris syndrome (IFIS), first described in 2005 , is characterized by the following triad of findings during cataract surgery: miosis, iris billowing during normal irrigation and aspiration in the anterior chamber, and iris prolapse into the phacoemulsification and side port incisions. ${ }^{1,2}$ IFIS not only increases the difficulty of cataract surgery but has also been associated with an increased risk of such surgical complications as retinal detachment, loss of lens fragments, endophthalmitis, and severe iris defects associated with pupil deformities. ${ }^{1,3,4}$ Several studies have reported the association between $\alpha$-adrenergic receptor antagonists and an increased risk of IFIS. ${ }^{1,5-7}$ Urologists and primary care providers prescribe $\alpha$-adrenergic blockers for the management of urinary tract symptoms associated with nephrolithiasis, chronic prostatitis, and benign prostate hyperplasia (BPH). ${ }^{8-10}$ The incidence of both $\mathrm{BPH}$ and cataracts increases with age, with $\sim 50 \%$ of men older than 65 years developing both cataracts and BPH as comorbidities. ${ }^{11}$ Alpha-adrenergic receptor antagonists are also used for prophylactic management of postoperative urinary retention. Studies have reported that the use of prophylactic $\alpha$-adrenergic antagonists by urologists reduces the incidence of postoperative urinary retention in patients undergoing a variety of scrotal, inguinal, penile, and perineal surgeries. ${ }^{12-14}$ By decreasing postoperative urinary retention, $\alpha$-adrenergic blockers decrease catheterization use, postoperative complications, health care costs, and length of hospital stays. ${ }^{13,14}$

Tamsulosin (Flomax) is the most commonly prescribed $\alpha$ blocker for BPH due to its 20-fold greater affinity for the $\alpha-1$ adrenergic receptor, located on the bladder-neck smooth muscle and the iris dilator muscle, rendering tamsulosin more uroselective and associated with fewer systemic side effects compared with other $\alpha$-adrenergic blockers. ${ }^{15}$ Due to its greater affinity for the $\alpha-1$ adrenergic receptor, tamsulosin has also been reported to have a stronger association (compared with other $\alpha$-adrenergic blockers) with IFIS and cataract surgery complications. ${ }^{1,3}$

In 2008, a survey sent to members of the American Society of Cataract and Refractive Surgery indicated that $95 \%$ of responders believed that taking $\alpha$-adrenergic blockers increases the risks associated with cataract surgery, and $91 \%$ believed that more education for prescribing physicians was necessary to ensure that prescribers would use $\alpha$ blockers with discretion. ${ }^{5}$ A 2016 study comprised of 175 American urologists indicated that $21 \%$ of responders never ask their patients about ophthalmologic conditions prior to prescribing $\alpha$ blockers and only 30\% counseled patients regarding an increased risk of IFIS with $\alpha$ blocker use. ${ }^{16}$ Additionally, a 2014 study showed that only $35 \%$ of primary care providers in the San Francisco Bay Area surveyed were aware of the relationship between IFIS and $\alpha$ blockers. ${ }^{17}$

The purpose of this study is to investigate United States urology residency program directors' (1) awareness of the association between $\alpha$-adrenergic receptor antagonists and IFIS, and (2) practice patterns regarding the use of prophylactic $\alpha$-adrenergic receptor antagonists prior to surgery.

\section{Materials and Methods}

The study was granted an exemption by the Institutional Review Board of the Penn State College of Medicine. An email containing a description of the study, an invitation to participate in the study, and a secure link to the anonymous, webbased survey was sent to the program director (PD) of each of the 138 urology residency programs accredited by the Accreditation Council for Graduate Medical Education (ACGME) as identified using the ACGME list serve database (www.acgme. org). Reminder emails were sent to non-responding PDs weekly for 3 weeks. The questionnaire (see Supplementary Material) surveyed PDs' awareness of the association between $\alpha$-adrenergic receptor antagonists and IFIS, and their practice patterns regarding the use of prophylactic a blockers prior to surgery.

\section{Results}

Sixty-nine (50\%) of the 138 urology PDs responded to the survey. On average, these urologists had been in practice for 18.3 years, with the majority performing between 200 to 500 surgeries per year. Thirty-six (52\%) respondents typically prescribe or consider prescribing prophylactic $\alpha$-adrenergic blockers before surgery. When asked their specific $\alpha$ blocker preference, 40 urologists responded and almost all (39, 98\%) preferred the use of the uroselective tamsulosin with 1 (2.5\%) preferring silodosin, another uroselective $\alpha-1$ adrenergic antagonist. Forty percent of respondents do not believe that tamsulosin increases the risk of IFIS.

The next stage of the survey assessed the prescribing patterns and influencing factors of the urology PDs who typically prescribe surgical prophylactic $\alpha$ blockers $(n=36)$. When asked about factors which increase the likelihood of them prescribing prophylactic $\alpha$ blockers prior to surgery, the urology PDs indicated the three most important factors were male gender of the patient $(30,83 \%)$, certain types of surgical procedures $(30,83 \%)$, and a patient being older than 55 years $(25,69 \%)$. The least considered factor was cataract surgery status of a patient, with only 2 (5.6\%) considering cataract surgery history when prescribing. Of the 36 urology PDs who prescribe prophylactic $\alpha$ blockers, 16 (44\%) ask their patients about their cataract history most ( $>50 \%$ ) of the time, while 10 (28\%) never ask. Additionally, 20 (56\%) would consider changing their $\alpha$-adrenergic antagonist prescription (lower dose, alternative drug, or not prescribing) upon learning that a patient has not had cataract surgery performed in both eyes. Upon prescribing a prophylactic $\alpha$ blocker, 21 (58\%) will counsel most $(>50 \%$ ) of their patients regarding the association between the prescribed $\alpha$ blocker and IFIS.

\section{Discussion}

In the current study, over half of the urology residency PDs typically prescribe or consider prescribing prophylactic $\alpha$ adrenergic blockers before surgery, with the uroselective drug tamsulosin being the agent of choice. With the majority of participating urology residency PDs seeing 200 to 500 surgical patients per year, the estimated number of patients receiving prophylactic $\alpha$ blockers is high. 
The $\alpha$-adrenergic blocker prescribed most frequently by urologists is tamsulosin, which is characterized by a 40 -fold higher odds ratio for IFIS compared with other $\alpha$-adrenergic blockers. ${ }^{18}$ The reported incidence of IFIS is greater than $40 \%$ for patients exposed to tamsulosin, compared with the $0.6 \%$ to $3.7 \%$ estimated incidence of IFIS in the general population. ${ }^{2,19,20}$ of note, a recent study of over 400,000 cataract operations from 2003 to 2013 showed that the rate of adverse events associated with cataract surgery in general, and the rate of adverse events associated with IFIS specifically, have decreased over time in part due to safer, adaptive techniques to combat IFIS such as viscosurgical devices, mechanical pupil expansion devices, and intensive pharmacologic pupil dilation. ${ }^{3,21}$ Considering the decrease in the IFIS-associated complication rate, as well as the benefits of $\alpha$ blockers with respect to postoperative recovery, the risk-benefit ratio of prophylactic $\alpha$ antagonist usage warrants further exploration.

Over a decade ago, multiple investigators called for a greater understanding of IFIS by prescribers of $\alpha$ blockers and properly educating patients about $\alpha$ blocker induced IFIS, such that patients inform their ophthalmologists of $\alpha$ blocker usage prior to cataract surgery to reduce the risk of complications. ${ }^{1,3,5,6}$ Our study results indicate that, over a decade later, greater education of, and awareness among, urologists regarding the association between $\alpha$ blockers and IFIS is still warranted. Forty percent of our urology PD respondents do not believe that tamsulosin increases the risk of IFIS, even though IFIS is strongly associated with tamsulosin and has been reported after a single dose of tamsulosin. ${ }^{1,3,15}$ Nearly half of the prescribing urologists surveyed do not counsel their patients regarding the association between $\alpha$ blockers and IFIS. This paucity of patient counseling makes it less likely for cataract surgeons to be informed of patients' perioperative $\alpha$ blocker usage, potentially leading to less use of the adaptive techniques for IFIS which have lowered adverse events to $1.0 \%$ of surgeries for tamsulosin users. ${ }^{3,21}$

Study limitations include the $50 \%$ response rate and the fact that our sample population included only urology residency PDs. Despite having a wide geographic distribution, our survey respondents may not be representative of the more general urologist population in America, especially regarding their knowledge about IFIS. A 2014 study indicated that the most common ways prescribers learned about IFIS was through medical journals and ophthalmologist colleagues. ${ }^{16}$ As these urology residency directors work in predominantly academic medical centers, we may expect the participants in this study to be more informed about IFIS than the general urologist population due to working with ophthalmologist colleagues and staying current with medical literature. The study population we selected plays an influential role in teaching future urologists and, therefore, represents an important group of urologists to target with respect to educational initiatives regarding the association between $\alpha$-blocking agents and IFIS.

\footnotetext{
Funding

None.

Conflict of Interest

None declared.
}

\section{References}

1 Chang DF, Campbell JR. Intraoperative floppy iris syndrome associated with tamsulosin. J Cataract Refract Surg 2005;31 (04):664-673

2 Yaycioglu O, Altan-Yaycioglu R. Intraoperative floppy iris syndrome: facts for the urologist. Urology 2010;76(02):272-276

3 Chang DF, Braga-Mele R, Mamalis N, et al; ASCRS Cataract Clinical Committee. ASCRS White Paper: clinical review of intraoperative floppy-iris syndrome. J Cataract Refract Surg 2008;34(12): 2153-2162

4 Takmaz T, Can I. Clinical features, complications, and incidence of intraoperative floppy iris syndrome in patients taking tamsulosin. Eur J Ophthalmol 2007;17(06):909-913

5 Chang DF, Braga-Mele R, Mamalis N, et al; ASCRS Cataract Clinical Committee. Clinical experience with intraoperative floppy-iris syndrome. Results of the 2008 ASCRS member survey. J Cataract Refract Surg 2008;34(07):1201-1209

6 Chadha V, Borooah S, Tey A, Styles C, Singh J. Floppy iris behaviour during cataract surgery: associations and variations. $\mathrm{Br} \mathrm{J}$ Ophthalmol 2007;91(01):40-42

7 Oshika T, Ohashi Y, Inamura M, et al. Incidence of intraoperative floppy iris syndrome in patients on either systemic or topical alpha(1)-adrenoceptor antagonist. Am J Ophthalmol 2007;143 (01):150-151

8 Wei JT, Calhoun E, Jacobsen SJ. Urologic diseases in America project: benign prostatic hyperplasia. J Urol 2008;179(5, Suppl):S75-S80

9 Nickel JC, Touma N. $\alpha$-Blockers for the treatment of chronic prostatitis/chronic pelvic pain syndrome: an update on current clinical evidence. Rev Urol 2012;14(3-4):56-64

10 Lipkin M, Shah O. The use of alpha-blockers for the treatment of nephrolithiasis. Rev Urol 2006;8(Suppl 4):S35-S42

11 Jan Teper S, Dobrowolski D, Wylegala E. Complications of cataract surgery in patients with BPH treated with alpha $1 \mathrm{~A}$-blockers. Cent European J Urol 2011;64(02):62-66

12 Akkoc A, Aydin C, Topaktas R, et al. Prophylactic effects of alphablockers, Tamsulosin and Alfuzosin, on postoperative urinary retention in male patients undergoing urologic surgery under spinal anaesthesia. Int Braz J Urol 2016;42(03):578-584

13 Mohammadi-Fallah M, Hamedanchi S, Tayyebi-Azar A. Preventive effect of tamsulosin on postoperative urinary retention. Korean J Urol 2012;53(06):419-423

14 Madani AH, Aval HB, Mokhtari G, et al. Effectiveness of tamsulosin in prevention of post-operative urinary retention: a randomized double-blind placebo-controlled study. Int Braz J Urol 2014;40(01):30-36

15 Foglar R, Shibata K, Horie K, Hirasawa A, Tsujimoto G. Use of recombinant alpha 1-adrenoceptors to characterize subtype selectivity of drugs for the treatment of prostatic hypertrophy. Eur J Pharmacol 1995;288(02):201-207

16 Zhang Y, Shamie N, Daneshmand S. Assessment of urologists' knowledge of intraoperative floppy iris syndrome. Urology 2016; 97:40-45

17 Doss EL, Potter MB, Chang DF. Awareness of intraoperative floppyiris syndrome among primary care physicians. J Cataract Refract Surg 2014;40(04):679-680

18 Chatziralli IP, Sergentanis TN. Risk factors for intraoperative floppy iris syndrome: a meta-analysis. Ophthalmology 2011; 118(04):730-735

19 Chang DF, Osher RH, Wang L, Koch DD. Prospective multicenter evaluation of cataract surgery in patients taking tamsulosin (Flomax). Ophthalmology 2007;114(05):957-964

20 Keklikci U, Isen K, Unlu K, Celik Y, Karahan M. Incidence, clinical findings and management of intraoperative floppy iris syndrome associated with tamsulosin. Acta Ophthalmol 2009;87(03):306-309

21 Campbell RJ, El-Defrawy SR, Gill SS, et al. Evolution in the risk of cataract surgical complications among patients exposed to tamsulosin: a population-based study. Ophthalmology 2019;126 (04):490-496 